\title{
The use of selective splenic vascular control in laparoscopic splenic vessels and spleen preservation distal pancreatectomy
}

\author{
Zhe Liu, Zhihuan Xiao, Guichen Li, Anjiang Gou, Yuanhong Xu, Shaowei Song, Kejian Guo, Gang Ma \\ Department of Pancreatic-Biliary Surgery, First Hospital of China Medical University, Shenyang, China \\ Contributions: (I) Conception and design: G Ma; (II) Administrative support: Z Liu; (III) Provision of study materials or patients: G Ma; \\ (IV) Collection and assembly of data: Z Xiao; (V) Data analysis and interpretation: A Gou; (VI) Manuscript writing: All authors; (VII) Final approval \\ of manuscript: All authors. \\ Correspondence to: Dr. Gang Ma. Department of Pancreatic-Biliary Surgery, First Hospital of China Medical University, 155 North Nanjing St., \\ Shenyang 110001, China. Email: magangzy@163.com.
}

\begin{abstract}
Backgroundin In the process of laparoscopic splenic vessels and spleen preservation distal pancreatectomy (LsvSPDP), because the splenic blood vessels have many small branches, how to safely separate the splenic blood vessels from the pancreas has always been the focus and difficulty of this operation. Many cases were switched to laparotomy, or the Warshaw method due to the inability to control bleeding during the separation of the splenic blood vessels. Therefore, we tried to use the selective splenic vascular control method when separating the splenic blood vessels to observe its effect on the conditions of the surgical patients during and after the operation.

Methods: We retrospectively collected 35 cases of LsvSPDP conducted in our center from September 2015 to December 2020, including 5 males and 30 females. Considering the influence of the surgical learning curve, the cases were divided into three groups. Finally, through statistics of its intraoperative and postoperative conditions, the effectiveness of selective splenic vascular control method can be judged.

Results: Patients in Group 2 and 3 showed significantly less blood loss (172.5 and $134.44 \mathrm{~mL}$, respectively) compared to patients in Group $1(541.43 \mathrm{~mL} ; \mathrm{P}=0.01)$. However, the amount of blood loss was not significantly different between Group 2 and 3.

Conclusions: The amount of bleeding was significantly reduced by splenic blood vessel control technology. And it can improve the success rate of spleen preservation, preserve the success rate of splenic blood vessels, and reduce intraoperative bleeding.
\end{abstract}

Keywords: Selective splenic vascular control; novel technique; laparoscopic splenic vessels and spleen preservation distal pancreatectomy (LsvSPDP)

Submitted Jun 17, 2021. Accepted for publication Aug 16, 2021.

doi: $10.21037 /$ gs-21-513

View this article at: https://dx.doi.org/10.21037/gs-21-513

\section{Introduction}

Since the implementation of the first case of laparoscopic splenic vessels and spleen preservation distal pancreatectomy (LsvSPDP) (1), it has become the treatment of choice for benign or low-grade malignant tumors located in the tail of the pancreas. This method is minimally invasive, preserves splenic function, and avoids the risk of hypoperfusion of the splenic artery associated with the Warshaw method. In this latter technique, resection of the splenic vein may cause splenic infarction and perigastric varices (2-8).

During LsvSPDP, the separation of the splenic arteries and veins from the pancreatic parenchyma, and their subsequent preservation, is of vital importance. However, due to the abundant blood supply of the pancreas, there are many branching blood vessels between the splenic blood vessels and the pancreatic parenchyma which increases the risk of intraoperative bleeding. Intraoperative hemorrhages 
Table 1 Patient characteristics

\begin{tabular}{|c|c|c|c|c|}
\hline Items & Group $1(n=14)$ & Group $2(n=12)$ & Group $3(n=9)$ & $P$ \\
\hline Gender & 14 & 12 & 9 & 0.576 \\
\hline Male & 1 & 2 & 2 & \\
\hline Female & 13 & 10 & 7 & \\
\hline Tumor size & $4.39 \pm 2.11$ & $4.17 \pm 1.7336$ & $3.61 \pm 1.833$ & 0.635 \\
\hline Histological diagnosis & & & & 0.251 \\
\hline SCN & 3 & 7 & 2 & \\
\hline MCN & 2 & 2 & 2 & \\
\hline PNET & 3 & 0 & 1 & \\
\hline Pancreatic pseudocyst & 1 & 1 & 2 & \\
\hline ACA & 0 & 1 & 0 & \\
\hline
\end{tabular}

BMI, body mass index; SCN, serous cystic neoplasm; MCN, mucinous cystic neoplasm; SPN, solid pseudopapillary neoplasm; IPMN, intraductal papillary mucinous neoplasms; PNET, pancreatic neuroendocrine tumors; ACA, acinar cell cystadenoma.

obscure the operation field and hinders the operation. If the bleeding cannot be stopped promptly and properly, the vascular breach will be torn and enlarged, and the bleeding will deteriorate. Eventually, it will be necessary to switch to laparotomy, or the Warshaw method, or a splenectomy $(9,10)$, leading to failure of LsvSPDP surgery. Therefore, the efficient and effective treatment of intraoperative bleeding is crucial for the successful implementation of LsvSPDP and successful tumor resection.

To date, there have been few studies examining the control of hemorrhage during minimally invasive pancreatic resection (MIPR) (3). This report details our own experience for controlling intraoperative hemorrhage during MIPR, which effectively reduced bleeding, preserved the integrity of the splenic blood vessels, and improved the success rate of spleen preservation surgery.

We present the following article in accordance with the STROBE reporting checklist (available at https://dx.doi. org/10.21037/gs-21-513).

\section{Methods}

The study was conducted in accordance with the Declaration of Helsinki (as revised in 2013). Ethical approval for this study was obtained from the Ethical Committee of the First Hospital of China Medical University (No. [2015]100). The patients provided their written informed consent to participate in this study.

\section{Patients}

This study retrospectively collected 35 cases of LsvSPDP conducted in our center from September 2015 to December 2020, including 5 males and 30 females. The specific criteria for inclusion included the initial diagnosis of benign pancreatic tumor, borderline pancreatic tumor, or lowgrade malignant tumor. Table 1 lists the specific pathological types and general conditions.

Considering the influence of the surgical learning curve (11), the cases were divided into three groups. Group 1 consisted of 14 cases between September 2015 and December 2018, with less than 5 cases per year. The traditional method was used and these cases are classified as "medium" on the learning curve. Group 2 consisted of 12 cases from January 2019 to December 2019. These cases used traditional methods and are considered to be past the learning curve. Group 3 consisted of 9 cases from January 2020 to December 2020. These cases used splenic 
blood vessel control technology.

\section{Procedures}

\section{Position}

Patients are placed in the reverse Trendelenburg, split leg position, with the left side elevated. Using the five-hole method, a $12 \mathrm{~mm}$ trocar is placed under the umbilicus and a light source is established. The surgeon is positioned on the right side of the patient, and the assistant is on the left side of the patient.

\section{Traditional method}

The left outer lobe of liver is suspended, the gastrocolonic ligament is cut, and the pancreas is exposed. The number and location of the lesions is confirmed. Taking the lateralposterior approach, the back of the pancreas is freed along the Told gap, and the body and tail of the pancreas is freed from the posterior abdominal wall. At $1 \mathrm{~cm}$ from the proximal end of the lesion, the splenic artery and vein are separated, and the posterior pancreatic tunnel is opened. The pancreas is cut off with the Johnson \& Johnson Echelon cutting and closing device. The distal pancreas is lifted and the splenic arteries and veins are separated from the pancreas.

\section{Splenic blood vessel control technology}

The blood vessel suture method was implemented to prevent possible splenic blood vessel bleeds. It can be used to immediately block the far and near ends of the bleeding blood vessel.

After separating the tail of the pancreas from the posterior abdominal wall, the tail of the pancreas is dissected and separated to expose the arteries and veins of the spleen. In the event of a bleed from the splenic blood vessel, a bulldog clamp can be used to block the splenic blood vessel on the side of the spleen. After separating the splenic arteries and veins at the proximal end, blocking bands are preset, and the blood vessels are separated from the pancreatic parenchyma by pulling and traction. In the event of a splenic blood vessel bleed, the blood vessel can be temporarily blocked from the proximal side.

\section{Statistical analysis}

Continuous data are expressed as mean and standard deviation. Categorical variables were analyzed with the chisquared test or Fisher exact test, and continuous variables were analyzed with the $t$-test. The comparison between two groups was performed using the Student-Newman-Keuls test. Results were considered statistically significant when $\mathrm{P}<0.05$. SPSS version 15.0 for Windows (SPSS, Chicago, IL, USA) was used for all analyses.

\section{Results}

Patients in Group 2 and 3 showed significantly less blood loss (172.5 and $134.44 \mathrm{~mL}$, respectively) compared to patients in Group $1(541.43 \mathrm{~mL} ; \mathrm{P}=0.01)$. However, the amount of blood loss was not significantly different between Group 2 and 3. The comparison of the average values showed that the amount of bleeding was reduced by splenic blood vessel control technology. All other clinical parameters were not significantly different among the three groups (Table 2).

\section{Discussion}

There are numerous advantages to using the selective splenic blood vessel control technique during LsvSPDP. The pancreas is located deep behind the peritoneum and the surface is covered by the stomach and the colon. When the gastric and colon ligaments are separated and the stomach and colon are pulled up and down, the pancreas is exposed (Figure 1). The anatomical structure of the body and tail of the pancreas becomes obvious with the pancreatic parenchyma composed of yellow lobules running horizontally, and the presence of two accompanying thick blood vessels (the splenic artery and vein). Such anatomical structure facilitates the exposure of the laparoscope and does not require frequent replacement of positions. Coupled with the magnification of the laparoscope, the fine anatomical structure between the pancreatic parenchyma and the splenic blood vessels can be clearly observed, which is conducive to the separation and protection of blood vessels.

Bleeding is one of the biggest challenges of laparoscopic pancreatic body and tail surgery (12). Effective control of the hemorrhage is the key to successful implementation of the operation. Although the hemostatic function of the ultrasonic scalpel has been greatly improved, the process of separating the splenic blood vessel from the pancreatic parenchyma is difficult. The splenic blood vessel often sends out many small branches into the pancreatic parenchyma and thus, the operating space is narrow. The head of ultrasonic scalpel may not be able to completely 
Table 2 Perioperative outcomes

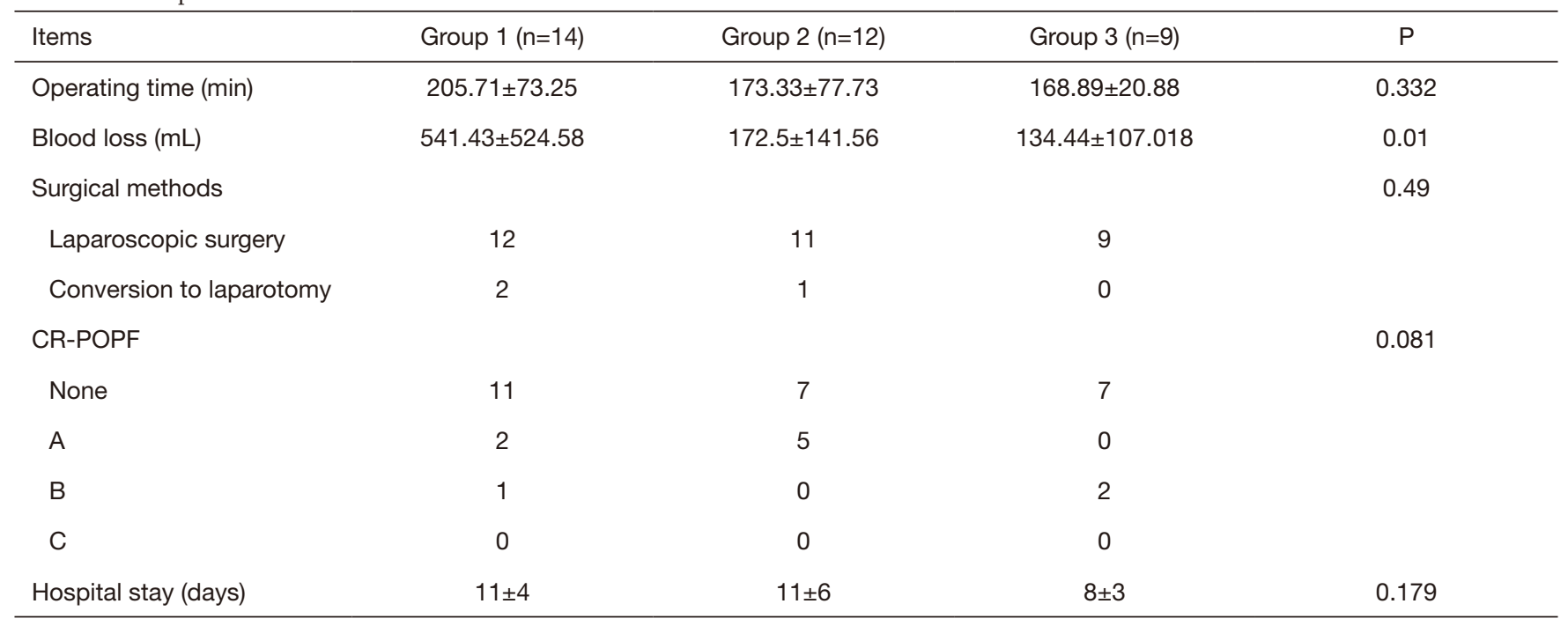

CR-POPF, clinically relevant postoperative pancreatic fistula.
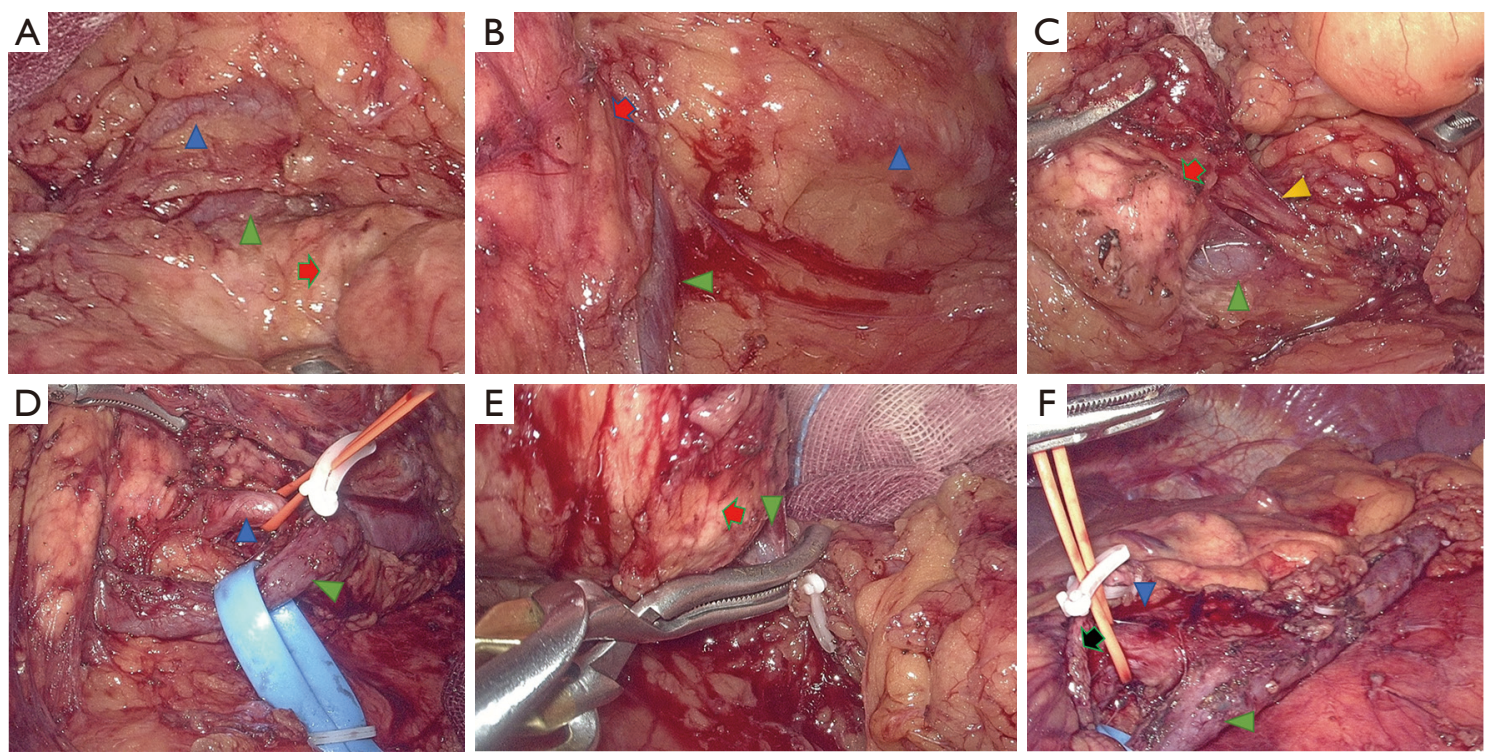

Figure 1 Intraoperative pictures. (A) Separate the splenic veins and artery near the hilum of spleen. (B) Lift the pancreatic tail, reveal the splenic artery and vein. (C) A splenic vein branches is plainly visible to the tail of pancreas. (D) Separate the spleen veins and artery under the neck of the pancreas. (E) A bulldog clamp can be placed through the distal splenic vein when splenic venous hemorrhage. (F) After pancreatectomy. Blue arrow: splenic artery; green arrow: splenic vein; red arrow: pancreatic tail; yellow arrow: a splenic vein branches to the tail of pancreas; black arrow: pancreatic head.

clamp the branches of the blood vessel, and it may not be able to effectively perform the clotting function, resulting in bleeding. In addition, even if the branch of the blood vessel has been coagulated, the blood vessel needs to be stretched during the separation process, which may cause the scab to fall off and cause bleeding. Therefore, it is especially important to effectively control any possible bleeds.

On an anatomical level, the proximal end of the splenic blood vessel is relatively easy to free because of the thicker blood vessels and fewer branches. The blood vessels on 
the hilar side of the spleen are also easy to free because the splenic artery is often separated from the pancreatic parenchyma when it travels to the hilum of the spleen. However, the splenic vein is closely related to the pancreatic parenchyma and can therefore represent a significant difficulty.

The splenic vein is the main pathway for blood returning from the spleen. Under normal circumstances (non-portal hypertension), the blood flow direction is from the splenic portal to the portal vein. When the branches of the splenic vein bleed, the splenic hilar blood flow is the main source of bleeding. Therefore, controlling the blood flow on the hilar side of the spleen controls most of the blood flow in the splenic vein. Thus, freeing the tail of the pancreas, and exposing and controlling the splenic vein on the hilar side of the spleen is the key to this method. In addition, the preferential separation of the splenic blood vessels on the hilar side of the spleen is also a necessary key step of the Warshaw method. This is an important step that must be performed when the tumor infiltrating the splenic blood vessels cannot be separated during the operation and the procedure must be converted to the Warshaw technique.

The tail of the pancreas is close to the hilum of the spleen, and the blood vessels at this point are often branches of the splenic vein adjacent to the tail of the pancreas. After the pancreas is freed from the posterior abdominal wall during the operation, the whole course of the splenic vein is exposed from the dorsal side of the pancreas. There may be a thin layer of pancreatic tissue covering the caudal side of the pancreas, and the pancreatic tissue needs to be cut along the surface of the blood vessel to expose it. After exposure, assessment of the branching of the splenic vein is necessary, including the number of branches, the location of the branches, and the positional relationship with the tumor. When the splenic vein is freed on the hilar side of the spleen, if the tumor goes to the hilar side of the spleen and exceeds the location of the branch of the splenic vein, the free dissection of the branches of the splenic vein needs to be performed at the distal end and pre-blocked.

During the dissection of the caudal side of the pancreas, accidental damage to the branches of the splenic vein may occur. At this time, the point of bleeding can be directly clamped, and the blood supply of the spleen will generally not be affected. If the tumor is large, located in the tail of the pancreas, close to the hilum of the spleen, or the tail of the pancreas extends into the hilum of the spleen and it is difficult to separate from the splenic blood vessels, the spleen may not be preserved and splenectomy is required.
In one case in this study, when the caudal blood vessel was separated, arterial branch bleeding occurred. Due to its small size, it could not be sutured and was instead clamped. As there were communicating branches between the splenic segments, and there was no ischemic manifestation.

The blood vessels on the cephalic side of the pancreas are first dissected and freed, and then the blocking bands are preset. This is different from direct blocking reported in other studies (13-15). There are numerous advantages to our method. First, ischemia-reperfusion injury associated with direct blocking can be avoided. Second, from our experience, the splenic artery is often on the upper edge of the pancreas, running upwards and backwards, with a large gap between the pancreatic parenchyma, and it is relatively easy to dissociate. However, the veins are often embedded in the pancreatic parenchyma, forming a splenic vein notch on the dorsal side of the pancreas. Separation is relatively difficult and bleeding is more common. Therefore, selective treatment is required to avoid the risk of aneurysm due to unnecessary arterial clamping injury. Third, selective blocking is performed only when the blood vessel is bleeding, and blocking is not performed in the absence of bleeding. When the blood vessel is filled, the gap between the blood vessel and the pancreatic tissue is more easily exposed, which is conducive to anatomical separation. The fourth advantage is a psychological one, as the surgeon does not need to be overly cautious, and thus the operation is calmer and the operation time is reduced.

Of the 9 cases using splenic blood vessel control technology, 3 patients $(33.3 \%)$ experienced vascular hemorrhage and their vessels were blocked. In one case the main splenic artery was blocked, and in the other case, the main splenic vein was blocked. After blocking, sutures were successfully performed to stop the bleeding and the integrity of the splenic blood vessels was maintained. If splenic blood vessel control technology was not used, it is likely that the surgical procedure would have failed and conversion to other methods would have been required. Therefore, the implementation of this new technology has increased the success rate of the surgery.

In this article, there were three cases of conversion to laparotomy due to blood loss. One case was changed to laparotomy due to a lack of experience and poor bleeding control. The other two cases were converted to the Warshaw method and combined with splenectomy because the blood vessels were not controlled and flustered during bleeding, resulting in increased vascular breaks and uncontrollable hemorrhage. 
In this study cohort, after the learning curve had been passed using the traditional method, the surgical effect improved significantly. The operation time was shortened and intraoperative bleeding was reduced. After adopting the new splenic blood vessel control technology, the surgical efficacy was further improved, and intraoperative bleeding was further reduced compared with the traditional method, resulting in a significant increase in the success rate of the surgery. There were no differences in surgical complications such as pancreatic fistula and postoperative bleeding, among any of the three groups.

Therefore, splenic blood vessel control technology allows the effective management of intraoperative bleeds by changing a passive response to a pre-emptive active control, which is beneficial to reducing intraoperative hemorrhage and preserving the integrity of splenic blood vessels. However, this current study has certain shortcomings, and the conclusions should be interpreted with caution. First, this investigation was a retrospective study. The number of cases in each group was small, and the lack of large sample data is prone to bias which can affect the accuracy and reliability of the results. Second, in the actual operation, a certain technique of vascular freeing is required. Sometimes during this part of the operation, branch blood vessels may bleed, which affects the completion of the operation. Third, in the selection of patients, although the spleenpreserving pancreatic body and tail resection is the first choice treatment for benign or borderline tumors of the body and tail of the pancreas (16), it is not necessarily suitable for all patients. Before surgery, it is important to consider computed tomography (CT) three-dimensional reconstruction, magnetic resonance imaging (MRI), and other imaging data to accurately determine the number and location of tumors, the relationship with the splenic blood vessels, the positional relationship between the pancreatic parenchyma and the splenic blood vessels, and the occurrence of regional portal hypertension if the splenic blood vessels are compressed or not visualized. In the case of tortuous expansion of the perisplenic blood vessels, pancreatitis, or a huge tumor in the tail of the pancreas, it will be difficult to separate the splenic blood vessels. In such cases, the Warshaw method or splenectomy may be appropriate $(17,18)$.

\section{Conclusions}

In conclusion, the splenic blood vessel control method is safe, effective, and simple to conduct. It can improve the success rate of spleen preservation, preserve the success rate of splenic blood vessels, and reduce intraoperative bleeding.

\section{Acknowledgments}

Funding: (I) Liaoning Provincial Department of Education science research project (L2014299); (II) National Natural Science Foundation of China (81572360).

\section{Footnote}

Reporting Checklist: The authors have completed the STROBE reporting checklist. Available at https://dx.doi. org/10.21037/gs-21-513

Data Sharing Statement: Available at https://dx.doi. org/10.21037/gs-21-513

Conflicts of Interest: All authors have completed the ICMJE uniform disclosure form (available at https://dx.doi. org/10.21037/gs-21-513). All authors report funding from Liaoning Provincial Department of Education science research project (L2014299) and National Natural Science Foundation of China (81572360). The authors have no other conflicts of interest to declare.

Ethical Statement: The authors are accountable for all aspects of the work in ensuring that questions related to the accuracy or integrity of any part of the work are appropriately investigated and resolved. The study was conducted in accordance with the Declaration of Helsinki (as revised in 2013). Ethical approval for this study was obtained from the Ethical Committee of the First Hospital of China Medical University (No. [2015]100). The patients provided their written informed consent to participate in this study.

Open Access Statement: This is an Open Access article distributed in accordance with the Creative Commons Attribution-NonCommercial-NoDerivs 4.0 International License (CC BY-NC-ND 4.0), which permits the noncommercial replication and distribution of the article with the strict proviso that no changes or edits are made and the original work is properly cited (including links to both the formal publication through the relevant DOI and the license). See: https://creativecommons.org/ licenses/by-nc-nd/4.0/. 


\section{References}

1. Jean-Philippe Adam, Alexandre Jacquin, Christophe Laurent, et al. Laparoscopic spleen-preserving distal pancreatectomy: splenic vessel preservation compared with the Warshaw technique. JAMA Surg 2013;148:246-52.

2. Melotti G, Butturini G, Piccoli M, et al. Laparoscopic distal pancreatectomy: results on a consecutive series of 58 patients. Ann Surg 2007;246:77-82.

3. Asbun HJ, Moekotte AL, Vissers FL, et al. The miami international evidence-based guidelines on minimally invasive pancreas resection. Ann Surg 2020;271:1-14.

4. Panda N, Bansal NK, Narsimhan M, et al. Spleenpreserving versus spleen-sacrificing distal pancreatectomy in laparoscopy and open method-perioperative outcome analysis-14 years experience. Indian J Surg 2016;78:90-5.

5. Tomulescu V, Copaescu C. Laparoscopic spleen preserving distal pancreatectomy with splenic vessels preservation. Chirurgia (Bucur) 2018;113:412-7.

6. Louis D, Alassiri A, Kirzin S, et al. Gastric bleeding risk following spleen preserving distal pancreatectomy with excision of the splenic vessels: a long-term follow-up. HPB (Oxford) 2017;19:345-51.

7. Blair AB, Sham JG. Spleen-preserving distal pancreatectomy with splenic vessel preservation: challenges in measuring the learning curve. Laparosc Surg 2018;2:56.

8. Huang J, Yadav DK, Xiong C, et al. Laparoscopic spleenpreserving distal pancreatectomy (LSPDP) versus open spleen-preserving distal pancreatectomy (OSPDP): a comparative study. Can J Gastroenterol Hepatol 2019;2019:9367868.

9. Jie Z, Hong L, Bin Z, et al. A new manoeuvre of vascular control in laparoscopic spleen-preserving distal pancreatectomy: Retrospective review for a modified Kimura's method. J Minim Access Surg 2021;17:43-8.

10. Juo YY, King JC. Robotic-assisted spleen preserving distal

Cite this article as: Liu Z, Xiao Z, Li G, Gou A, Xu Y, Song S, Guo K, Ma G. The use of selective splenic vascular control in laparoscopic splenic vessels and spleen preservation distal pancreatectomy. Gland Surg 2021;10(8):2528-2534. doi: 10.21037/ gs-21-513 pancreatectomy: a technical review. J Vis Surg 2017;3:139.

11. Kim HS, Park JS, Yoon DS. True learning curve of laparoscopic spleen-preserving distal pancreatectomy with splenic vessel preservation. Surg Endosc 2019;33:88-93.

12. Yoon YS, Lee KH, Han HS, et al. Effects of laparoscopic versus open surgery on splenic vessel patency after spleen and splenic vessel-preserving distal pancreatectomy: a retrospective multicenter study. Surg Endosc 2015;29:583-8.

13. Chen JH, Huang KF, Li CH. Preservation of splenic vessels during laparoscopic spleen-preserving distal pancreatectomy via lateral approach. Wideochir Inne Tech Maloinwazyjne 2015;10:382-8.

14. Nagakawa Y, Sahara Y, Hosokawa Y, et al. The straightened splenic vessels method improves surgical outcomes of laparoscopic distal pancreatectomy. Dig Surg 2017;34:289-97.

15. Hua YF, Yadav DK, Bai X, et al. Laparoscopic spleenpreserving distal pancreatectomy (LSPDP) with preservation of splenic vessels: an inferior-posterior approach. Gastroenterol Res Pract 2018;2018:1683719.

16. Zhang RC, Ma J, Mou YP, et al. Short- and long-term outcomes of laparoscopic organ-sparing resection for pancreatic neuroendocrine neoplasms. World J Surg 2020;44:3795-800.

17. Liao TK, Wang CJ, Su PJ, et al. Laparoscopic splenic vessels and spleen preservation distal pancreatectomy via inferior-posterior splenic vein approach. Surg Laparosc Endosc Percutan Tech 2020;30:424-9.

18. Nakamura Y, Matsushita A, Mizuguchi Y, et al. Study on laparoscopic spleen preserving distal pancreatectomy procedures comparing splenic vessel preservation and nonpreservation. Transl Gastroenterol Hepatol 2016;1:27.

(English Language Editor: J. Teoh) 